\title{
Epidemiology and risk factors of chronic kidney disease in India - results from the SEEK (Screening and Early Evaluation of Kidney Disease) study
}

Ajay K Singh ${ }^{1 *+}$, Youssef MK Farag ${ }^{1+}$, Bharati V Mittal ${ }^{1 \dagger}$, Kuyilan Karai Subramanian $^{1}$, Sai Ram Keithi Reddy ${ }^{1}$ Vidya N Acharya ${ }^{2}$, Alan F Almeida ${ }^{3}$, Anil Channakeshavamurthy ${ }^{4}$, H Sudarshan Ballal $^{5}$, Gaccione P ${ }^{1}$, Rajan Issacs ${ }^{6}$, Sanjiv Jasuja ${ }^{7}$, Ashok L Kirpalani ${ }^{8}$, Vijay Kher ${ }^{9}$, Gopesh K Modi ${ }^{10}$, Georgy Nainan ${ }^{11}$, Jai Prakash ${ }^{12}$, Devinder Singh Rana ${ }^{14}$, Rajanna Sreedhara ${ }^{4,15}$, Dilip Kumar Sinha ${ }^{16}$, Shah Bharat $V^{17}$, Sham Sunder ${ }^{18}$, Raj K Sharma ${ }^{19}$, Sridevi Seetharam ${ }^{4}$, Tatapudi Ravi Raju ${ }^{20}$ and Mohan M Rajapurkar ${ }^{13}$

\begin{abstract}
Background: There is a rising incidence of chronic kidney disease that is likely to pose major problems for both healthcare and the economy in future years. In India, it has been recently estimated that the age-adjusted incidence rate of ESRD to be 229 per million population (pmp), and >100,000 new patients enter renal replacement programs annually.

Methods: We cross-sectionally screened 6120 Indian subjects from 13 academic and private medical centers all over India. We obtained personal and medical history data through a specifically designed questionnaire. Blood and urine samples were collected.

Results: The total cohort included in this analysis is 5588 subjects. The mean \pm SD age of all participants was $45.22 \pm 15.2$ years (range 18-98 years) and $55.1 \%$ of them were males and $44.9 \%$ were females. The overall prevalence of CKD in the SEEK-India cohort was $17.2 \%$ with a mean eGFR of $84.27 \pm 76.46$ versus $116.94 \pm 44.65 \mathrm{~mL} / \mathrm{min} / 1.73 \mathrm{~m} 2$ in non-CKD group while 79.5\% in the CKD group had proteinuria. Prevalence of CKD stages 1, 2, 3, 4 and 5 was 7\%, $4.3 \%, 4.3 \%, 0.8 \%$ and $0.8 \%$, respectively.

Conclusion: The prevalence of CKD was observed to be $17.2 \%$ with $\sim 6 \%$ have CKD stage 3 or worse. CKD risk factors were similar to those reported in earlier studies.

It should be stressed to all primary care physicians taking care of hypertensive and diabetic patients to screen for early kidney damage. Early intervention may retard the progression of kidney disease. Planning for the preventive health policies and allocation of more resources for the treatment of CKD/ESRD patients are imperative in India.
\end{abstract}

Keywords: Chronic kidney disease, Prevalence, Epidemiology, Risk factors, SEEK, Screening programs, India, Diabetes, Hypertension, South East Asia

\footnotetext{
* Correspondence: asingh@partners.org

${ }^{\dagger}$ Equal contributors

'Brigham \& Women's Hospital \& Harvard Medical School, 75 Francis Street,

Boston, MA 02115, USA

Full list of author information is available at the end of the article
} 


\section{Background}

Chronic kidney disease (CKD) is emerging to be an important chronic disease globally [1]. One reason is the rapidly increasing worldwide incidence of diabetes [2] and hypertension [3,4]. In India, given its population $>1$ billion, the rising incidence of CKD is likely to pose major problems for both healthcare and the economy in future years. Indeed, it has been recently estimated that the age-adjusted incidence rate of ESRD in India to be 229 per million population (pmp) [5], and $>100,000$ new patients enter renal replacement programs annually in India [6]. On the other hand, because of scarce resources, only $10 \%$ of the Indian ESRD patients receive any renal replacement therapy (RRT) [6-8]. The lack of community-based screening programs has led to patients being detected with CKD at an advanced stage. It is possible that early detection of kidney disease through community based screening programs might have an impact on this problem through earlier intervention. The Screening and Early Evaluation of Kidney Disease Project (SEEK) was designed and performed to generate data to determine the prevalence and risk factors for CKD in India.

\section{Methods}

Thirteen academic and private medical centers in India participated in the study under the name of "Screening and Early Evaluation of Kidney disease - SEEK." Any Indian male and female with age over 18 years are eligible to participate in the screening. It was conducted between June 2005 to May 2007, coordinated from the Brigham and Women's Hospital in Boston, Massachusetts, a teaching affiliate of Harvard Medical School. The protocol was approved by the Partner's Institutional Review Board (IRB) as well as by individual centers own institutional IRBs. Signed or verbal informed consent (confirmed by a witness) was obtained before administering the questionnaire, taking measurements or blood collection. The database is based at the Brigham and Women's Hospital.

\section{Questionnaire}

A structured questionnaire was developed and pre-tested in the pilot study of 500 subjects carried out at one center in South India (Additional file 1). The questionnaire was translated into local languages (e.g., Telugu, Hindi, Marathi, Kannada, Gujarati and Malayalam). The questionnaire was generally administered by non-medical staff /volunteers who were trained by the SEEK-India team in interviewing techniques. At every site, staff was trained in interview techniques and measurement of height, weight and blood pressure by organizing a half day workshop prior to the camp. A team of nephrologists, nurses, technicians and trained interviewers participated in the camps. Questionnaires collected from the field were reviewed by the local site principal investigator and data entry was carried out locally.

\section{Anthropometric measures}

Body mass index (BMI) was calculated using the formula "weight (Kg) / height (m2)." The waist to hip circumference ratio (WHR) was calculated by using the waist circumference at the narrowest circumference between the lower costal margin and the iliac crest. Hip circumference was measured at the maximum circumference at the level of the femoral trochanters.

\section{Blood pressure measurement}

In order to get a standardized blood pressure (BP) measurement, a protocol per American Heart Association guidelines [9] and a power-point presentation was provided to the centers, and staff training was carried out prior to camps. Systolic blood pressure (SBP) was based on the 1st Korotkoff phase and diastolic (DBP) on the 5th Korotkoff phase. Mercury sphygmomanometer was used after checking for zero error. BP was recorded in the sitting position in the right arm supported at heart level, to the nearest $2 \mathrm{~mm}$ using mercury sphygmomanometer. An average of two readings was taken into consideration.

\section{Blood and Urine sample collection}

Random blood samples were collected. Blood was sent to a central laboratory. Quality control for temperature transporting specimens was checked and confirmed; i.e., 4-9 degree Celsius. Serum creatinine was measured using Jaffe Colorimetric method on a Roche Hitachi 912 analyzer. The instrument was calibrated (external calibration) using the Cleveland Clinic Foundation (CCF) creatinine panel. Regression analysis was carried out to calculate a formula to convert creatinine values obtained at the SRL-Ranbaxy laboratory (SRL) to the CCF values as follows: CCF creatinine $=-0.13+$ SRL creatinine $* 0.99$. Urine protein was detected by dipstick method (Bayer Multistix $10 \mathrm{SG}$ ). A modified MDRD-3 equation GFR $(\mathrm{mL} / \mathrm{min} / 1.73 \mathrm{~m} 2)=$ $175 \times(\mathrm{Scr})^{-1.54} \times(\text { Age })^{-0.203} \times(0.742$ if female $) \times(1.212$ if African American) was used [10]. Plasma glucose was measured by the glucose oxidase peroxidase method using Roche Hitachi 912 analyzer.

\section{Variables definitions}

Hypertension was defined as SBP/DBP $>=140 / 90 \mathrm{~mm}$ of $\mathrm{Hg}$ [11] or if the patient was on medication for hypertension or had a positive self reported history of hypertension (based on a response to "have you ever been told that you have high blood pressure" or "a past history of high blood pressure"). 
Diabetes was defined as fasting blood sugar FBS $>=126$ or random blood sugar $>=200$ or on any medications for diabetes mellitus (ADA definition) [12], or if there was a positive response to the questions "have you ever been told that you have diabetes" or "past history of diabetes." Self reported history of medications was verified and if the subject did not know the name of the medication and or if the stated name was incorrect, the response was considered as "no" even if the subject's response to the question on "are you on BP or diabetes medications" was "yes."

According to the International Diabetes Federation (IDF) criteria, abdominal obesity was defined waist circumference of $\geq 94 \mathrm{~cm}$ in men and $\geq 80 \mathrm{~cm}$ in women [13].

CKD stages were defined using NKF-KDOQI guidelines (eGFR $<60 \mathrm{ml} / \mathrm{min} / 1.73 \mathrm{~m} 2$ or proteinuria $>1+$ on dipstick) [14]. Urine protein positivity (proteinuria) was defined as urine albumin $1+$ or more. We used the CKD-EPI equation [15] in the sensitivity analysis to further explore the burden of CKD using this equation.

Self reported ischemic heart disease was taken as present if there was a self reported history of a myocardial infarction, percutaneous angioplasty or coronary artery bypass surgery (CABG).

All patients with CKD diagnosed in camps received the reports along with a referral to the local hospitals/clinics.

\section{Statistical analysis}

IBM SPSS Statistics for Windows, version 19, 2010, SPSS Inc., an IBM company, was used. In the descriptive analysis, continuous variables were expressed as mean \pm standard deviation (SD) and categorical variables were expressed as count (percentages). While making no assumption about the distribution of data, normality distribution testing of the continuous variables was performed using the non-parametric test; KolmogorovSmirnov test (KS-test). Univariate analyses comparing distributions of socio-demographic and clinical/historical measures between CKD groups was performed using independent unpaired student $t$ Test for normally distributed continuous variables and Mann-Whitney U Test for non-normally distributed continuous variables. For categorical variables we used Pearson chi-square test. Fisher's exact test was used when there was one or more of cells with an expected frequency $<5$. We performed spearman correlation analysis to study the relationship between eGFR and other variables. The values of Spearman's rho and the p-value have been reported.

\section{Results}

Six thousand one hundred and twenty subjects were screened as part of the SEEK-India project. We recruited subjects through 53 screening camps in 12 cities across India representing almost all Indian regions (Table 1). We excluded subjects who were less than 18 years of
Table 1 Distribution of subjects among screening centers and regions

\begin{tabular}{lc}
\hline Region and center & Number of subjects recruited \\
\hline North India & $\mathbf{2 2 9 8 ( 3 6 . 7 )}$ \\
Varanasi, Uttar Pradesh & $515(8.8 \%)$ \\
Kanpur, Uttar Pradesh & $511(9.1 \%)$ \\
Delhi & $752(10.9 \%)$ \\
Himachal Pradesh & $519(7.9 \%)$ \\
Northwest Inida & $\mathbf{4 0 2}(\mathbf{6 . 9 \% )}$ \\
Ludhiana, Punjab & $402(6.9 \%)$ \\
Central India & $\mathbf{4 3 8}(\mathbf{7 . 4 \% )}$ \\
Bhopal, Madhya Pradesh & $438(7.4 \%)$ \\
Western India & $\mathbf{1 0 3 7}(\mathbf{1 7 \% )}$ \\
Nadiad, Gujarat & $506(8.9 \%)$ \\
Bombay, Maharashtra & $531(8.1 \%)$ \\
Southwest India & $\mathbf{1 7 9 4 ( 2 9 . 5 \% )}$ \\
Mysore, Karnataka & $1022(17.1 \%)$ \\
Bangalore, Karnataka & $275(4.5 \%)$ \\
Cochin, Kerala & $497(7.9 \%)$ \\
Southeast India & $\mathbf{1 5 2 ( 2 . 5 \% )}$ \\
Visakhapatnam, Andhra Pradesh & $152(2.5 \%)$ \\
Total & $\mathbf{6 1 2 0}$ \\
\hline
\end{tabular}

age $(n=28)$, with history of dialysis $(n=19)$ and with history of kidney transplantation $(n=6)$ (Figure 1). We further excluded subjects for which certain variables' results were not recorded. These variables included gender, age, history of dialysis or kidney transplantation, serum creatinine and urine albumin. The total cohort included in this analysis is 5588 subjects. The mean \pm SD age of all participants was $45.22 \pm 15.2$ years (range 1898 years) and $55.1 \%$ of them were males and $44.9 \%$ were females. Hypertension was observed in $43.1 \%$ of our population while $18.8 \%$ of them were diabetic. The mean \pm SD of BMI was $23.91 \pm 5.3 \mathrm{~kg} / \mathrm{m} 2$. Defining overweight and obesity as BMI between $25-30$ and $>30 \mathrm{~kg} / \mathrm{m} 2$, respectively, the prevalence of overweight and obesity in our sample was $26.4 \%$ and $11.7 \%$, respectively. However, $36.5 \%$ had abdominal obesity where the mean waist circumference was $83.03 \pm 14 \mathrm{~cm}$. The mean estimated glomerular filtration rate (eGFR) using the MDRD-3 and CKD-EPI equations were $111.31 \pm 53$ and $104.9 \pm 25.52 \mathrm{~mL} / \mathrm{min} / 1.73 \mathrm{~m}^{2}$ respectively. The remaining baseline demographics, clinical and laboratory data were summarized in Tables 2 and 3.

\section{Prevalence of CKD}

Using MDRD equation, the overall prevalence of CKD in the SEEK-India cohort was $17.2 \%$ with a mean eGFR 


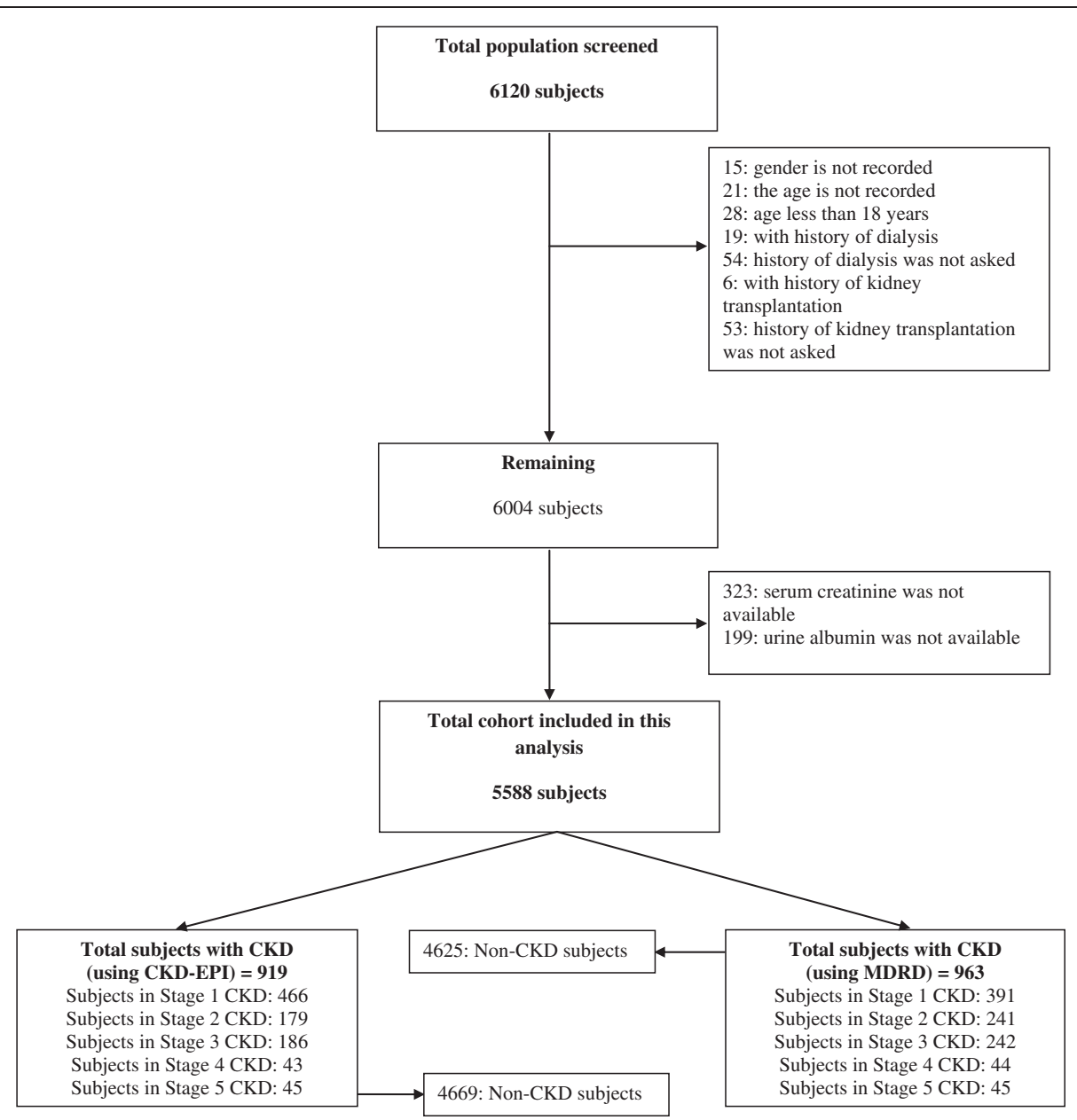

Figure 1 Flowchart of the SEEK-India Cohort.

of $84.27 \pm 76.46$ versus $116.94 \pm 44.65 \mathrm{~mL} / \mathrm{min} / 1.73 \mathrm{~m} 2$ in non-CKD group while $79.5 \%$ in the CKD group had proteinuria. Prevalence of CKD stages 1, 2, 3, 4 and 5 was $7 \%, 4.3 \%, 4.3 \%, 0.8 \%$ and $0.8 \%$, respectively (Figure 2).

CKD was higher in males across all stages of CKD (Figure 3). Those subjects who had low eGFR $(<60 \mathrm{ml} /$ $\mathrm{min} / 1.73 \mathrm{~m} 2)$ comprised $5.9 \%$ of the sample $(\mathrm{N}=331)$ while $13.7 \%$ had proteinuria $(\mathrm{N}=766)$.

The prevalence of CKD was center-dependent (Figure 4). The highest prevalence of CKD was observed in Visakhapatnam, Andhra Pradesh (46.8\%), Kanpur, Uttar Pradesh (41.7\%) and Delhi (41\%). The lowest prevalence was observed in Mysore and Bangalore in Karnataka state (4.2\% and $4 \%$, respectively).

\section{CKD Risk factors}

Patients with CKD were older, more likely to be male, more likely to have a high school diploma, more likely to be urban, less likely to have a low income, more likely to be overweight or obese, to have diabetes, hypertension and cardiovascular disease than patients without CKD. The most common risk factors and other characteristics among the subjects diagnosed with CKD were hypertension (64.5\%), anemia (40.7\%) and diabetes (31.6\%) (Figure 5). Anthropometric measures (except height), blood pressure, hemoglobin, random and fasting blood glucose correlated significantly with eGFR in the study cohort. However, age, blood pressure and hemoglobin correlated with eGFR in the CKD subgroup (Table 4).

Only $7.9 \%$ of the subjects with CKD were aware that they have CKD, while $5.9 \%$ of those with proteinuria reported knowing that they had protein in the urine.

\section{Sensitivity analysis using the CKD-EPI equation}

We used the CKD-EPI equation in a sensitivity analysis to test whether it produced similar results to the MDRD-3 equation (Tables 2 and 3). Using the CKD-EPI equation, the prevalence of CKD was $16.4 \%$. CKD Stage 1 was $8 \%$, slightly higher than the one estimated using MDRD-3. However, the prevalence of CKD stages 2 and 3 were $3.2 \%$ and $3.3 \%$, respectively and slightly lower 
Table 2 Baseline demographic and risk factors data for SEEK India Cohort

\begin{tabular}{|c|c|c|c|c|c|c|c|}
\hline & \multirow{2}{*}{$\begin{array}{c}\text { All } \\
\text { participants }\end{array}$} & \multicolumn{3}{|c|}{ By CKD status (using MDRD) } & \multicolumn{3}{|c|}{ By CKD status (using CKD-EPI) } \\
\hline & & CKD & Non-CKD & $p$ & CKD & Non-CKD & $p$ \\
\hline Age (in years) $(n=5588)$ & $45.22 \pm 15.2$ & $52.27 \pm 14.78$ & $43.75 \pm 14.88$ & $<0.0001$ & $52.19 \pm 14.9$ & $43.85 \pm 14.89$ & $<0.0001$ \\
\hline \multicolumn{8}{|l|}{ Gender } \\
\hline Male & $55.1 \%$ & $61 \%$ & $53.9 \%$ & $<0.0001$ & 62.5 & 53.7 & $<0.0001$ \\
\hline Female & $44.9 \%$ & $39 \%$ & $46.1 \%$ & & 37.5 & 46.3 & \\
\hline \multicolumn{8}{|l|}{ Education } \\
\hline$<$ High school & $43.6 \%$ & $37.6 \%$ & $45.1 \%$ & $<0.0001$ & 37.1 & 45.2 & $<0.0001$ \\
\hline$>=$ High school & $56.2 \%$ & $62.4 \%$ & $54.9 \%$ & & 62.9 & 54.8 & \\
\hline Residence status (urban) & $49.7 \%$ & $73.4 \%$ & $44.7 \%$ & $<0.0001$ & & & \\
\hline Income ( $<\$ 125 /$ month) & $63.7 \%$ & $52.6 \%$ & $69 \%$ & $<0.0001$ & & & \\
\hline Present or Past Smoker & $19.9 \%$ & $21.2 \%$ & $20 \%$ & 0.227 & 21.5 & 20 & 0.158 \\
\hline Overweight (BMI $25-30 \mathrm{~kg} / \mathrm{m}^{2}$ ) & $26.4 \%$ & $31.6 \%$ & $25.5 \%$ & $<0.0001$ & $31.6 \%$ & $25.5 \%$ & $<0.0001$ \\
\hline Obesity (BMI > = $30 \mathrm{~kg} / \mathrm{m}^{2}$ ) & $11.7 \%$ & $16.8 \%$ & $10.7 \%$ & $<0.0001$ & 16.6 & 10.8 & $<0.0001$ \\
\hline Abdominal obesity & $36.5 \%$ & $48.1 \%$ & $36.4 \%$ & $<0.0001$ & $47.8 \%$ & $36.6 \%$ & $<0.0001$ \\
\hline Diabetes & $18.8 \%$ & $31.6 \%$ & $16.1 \%$ & 0.000 & 32.2 & 16.1 & $<0.0001$ \\
\hline HTN & $43.1 \%$ & $64.5 \%$ & $41.9 \%$ & $<0.0001$ & 64.6 & 42.1 & $<0.0001$ \\
\hline Anemia & $33.1 \%$ & $40.7 \%$ & $31.8 \%$ & $<0.0001$ & 41.1 & 31.8 & $<0.0001$ \\
\hline History of Ischemic heart disease & 5.2 & 6.9 & 4.8 & 0.007 & 7 & 4.8 & 0.006 \\
\hline History of stroke & $1.3 \%$ & $2.6 \%$ & $1 \%$ & $<0.0001$ & 2.5 & 1 & 0.001 \\
\hline History of Hypercholesterolemia & $5.3 \%$ & 7.7 & 4.8 & $<0.0001$ & 7.6 & 4.8 & 0.001 \\
\hline History of PVD & $2.4 \%$ & 3 & 2.9 & 0.847 & 3 & 2.9 & 0.973 \\
\hline History of TB & $4.6 \%$ & 3.1 & 4.9 & 0.015 & 3.3 & 4.9 & 0.033 \\
\hline History of kidney stones & 4.5 & 5.3 & 4.3 & 0.196 & 5.2 & 4.4 & 0.254 \\
\hline
\end{tabular}

Diabetes: $\mathrm{FBS}>=126 \mathrm{mg} / \mathrm{dL}$ or RBS $>=200 \mathrm{mg} / \mathrm{dL}$ or self-reported or positive medication for diabetes confirmed by a physician.

HTN: $B P>=140 / 90 \mathrm{mmHg}$ or self-reported or positive medication for hypertension confirmed by a physician.

Anemia: NKF definition: hemoglobin level $<13.5 \mathrm{~g} / \mathrm{dl}$ in men and $12.0 \mathrm{~g} / \mathrm{dl}$ in women.

IHD: self-reported history of IHD or angioplasty or CABG.

PVD: Peripheral Vascular Disease.

than MDRD. The CKD-EPI equation performed similar to MDRD-3 in CKD stages 4 and 5 (0.8\% each). A comparison between the CKD and non-CKD subgroups (as defined using the CKD-EPI equation) with respect to the baseline demographics, clinical and laboratory variables, did not yield different results.

\section{Discussion}

The main finding of this study using a convenience cohort design is that the prevalence of CKD in the SEEKIndia cohort is $17.2 \%$. The prevalence of CKD stages 1 , $2,3,4$ and 5 was $7 \%, 4.3 \%, 4.3 \%, 0.8 \%$ and $0.8 \%$, respectively. Hypertension, anemia and diabetes were the most common risk factors and associated characteristics associated with CKD. Furthermore, old age, hypertension (both SBP and DBP) and low hemoglobin level were correlated with decreased eGFR in the CKD subgroup.

A recent study on 3398 central government employees in India reported $~ 13-15 \%$ prevalence of early stages of CKD (Stages 1, 2 and 3), similar in magnitude to our results, although they used microproteinuria, hematuria and/or leukocyturia as indicators of kidney damage. A study from Apollo Hospital in Chennai [16] reported a prevalence of impaired renal function (eGFR less than $80 \mathrm{~mL} / \mathrm{min} . \mathrm{m} 2$ ) as $0.86 \%$ to $1.39 \%$. This was done through the Rural Program of The Kidney Help Trust of Chennai. The investigators applied a regular screening program of an entire population of 25,000 while treating patients for diabetes and hypertension. Although the Chennai study was a population-based design, it was restricted only residents of a group of villages and hamlets about $50 \mathrm{~km}$ away from Chennai [17]. This makes generalization to the entire Indian population difficult. Furthermore, they did not include in their definition of CKD those subjects with proteinuria. In our SEEK-India cohort, $79.5 \%$ of those subjects diagnosed with CKD had proteinuria.

Agrawal et al. [18] performed a community-based study to determine the prevalence of CKD in the South Zones of Delhi. They used the multi-stage cluster 
Table 3 Clinical and laboratory data for the SEEK India cohort

\begin{tabular}{|c|c|c|c|c|c|c|c|}
\hline & \multirow{2}{*}{$\begin{array}{c}\text { All } \\
\text { participants }\end{array}$} & \multicolumn{2}{|c|}{ By CKD status (using MDRD) } & \multirow[b]{2}{*}{$p$} & \multicolumn{3}{|c|}{ By CKD status (using CKD-EPI) } \\
\hline & & CKD & Non-CKD & & CKD & Non-CKD & $p$ \\
\hline Weight (kg) & $60.74 \pm 14.3$ & $63.15 \pm 14.68$ & $60.24 \pm 14.2$ & $<0.0001$ & $63.26 \pm 14.75$ & $60.24 \pm 14.19$ & $<0.0001$ \\
\hline Height $(\mathrm{cm})$ & $159.4 \pm 10.3$ & $158.7 \pm 10.2$ & $159.52 \pm 10.3$ & 0.062 & $158.87 \pm 10.9$ & $159.47 \pm 10.32$ & 0.276 \\
\hline BMI $\left(\mathrm{kg} / \mathrm{m}^{2}\right)$ & $23.91 \pm 5.3$ & $25.12 \pm 5.67$ & $23.66 \pm 5.2$ & $<0.0001$ & $25.1 \pm 5.69$ & $23.68 \pm 5.2$ & $<0.0001$ \\
\hline Waist circumference $(\mathrm{cm})$ & $83.03 \pm 14$ & $87.01 \pm 14.4$ & $82.26 \pm 13.8$ & $<0.0001$ & $86.96 \pm 14.58$ & $82.31 \pm 13.78$ & $<0.0001$ \\
\hline Hip circumference $(\mathrm{cm})$ & $92.31 \pm 12$ & $94.43 \pm 12.36$ & $91.9 \pm 11.9$ & $<0.0001$ & $94.39 \pm 12.41$ & $91.93 \pm 11.88$ & $<0.0001$ \\
\hline WH ratio & $0.9 \pm 0.09$ & $0.92 \pm 0.09$ & $0.89 \pm 0.089$ & $<0.0001$ & $0.92 \pm 0.092$ & $0.89 \pm 0.089$ & $<0.0001$ \\
\hline Average SBP $(\mathrm{mmHg})$ & $126.63 \pm 19.69$ & $134.45 \pm 22.12$ & $125 \pm 18.75$ & $<0.0001$ & $134.51 \pm 22.1$ & $125.08 \pm 18.81$ & $<0.0001$ \\
\hline Average $\mathrm{DBP}(\mathrm{mmHg})$ & $80.32 \pm 11.22$ & $83.89 \pm 11.91$ & $79.59 \pm 10.94$ & $<0.0001$ & $83.89 \pm 11.92$ & $79.62 \pm 10.96$ & $<0.0001$ \\
\hline Hemoglobin $(g / d l)$ & $13.21 \pm 1.96$ & $12.94 \pm 2.2$ & $13.27 \pm 1.9$ & 0.001 & $12.94 \pm 2.2$ & $13.27 \pm 1.91$ & 0.001 \\
\hline Fasting blood glucose (mg/dL) & $107.54 \pm 46.23$ & $130.65 \pm 74.39$ & $102.61 \pm 35.95$ & 0.071 & $130.45 \pm 75.1$ & $102.76 \pm 35.96$ & 0.109 \\
\hline Random blood glucose (mg/dL) & $116.59 \pm 59.24$ & $131.94 \pm 71.8$ & $113.44 \pm 55.8$ & $<0.0001$ & $132.62 \pm 72.46$ & $113.49 \pm 55.82$ & $<0.0001$ \\
\hline Serum creatinine $(\mathrm{mg} / \mathrm{dL})$ & $0.82 \pm 0.78$ & $1.33 \pm 1.73$ & $0.71 \pm 0.19$ & $<0.0001$ & $1.34 \pm 1.77$ & $0.71 \pm 0.19$ & $<0.0001$ \\
\hline eGFR (MDRD) (mL/min/1.73 $\left.\mathrm{m}^{2}\right)$ & $111.31 \pm 53$ & $84.27 \pm 76.46$ & $116.94 \pm 44.65$ & $<0.0001$ & - & - & - \\
\hline eGFR (CKD-EPI) $\left(\mathrm{mL} / \mathrm{min} / 1.73 \mathrm{~m}^{2}\right)$ & $104.9 \pm 25.52$ & - & - & - & $82.25 \pm 34.95$ & $109.37 \pm 20.45$ & $<0.0001$ \\
\hline Urine protein (\%) & 13.7 & 79.5 & 0 & $<0.0001$ & 83.4 & 0 & $<0.0001$ \\
\hline Hematuria (\%) & 19 & 29.6 & 16.9 & $<0.0001$ & 30.4 & 16.9 & $<0.0001$ \\
\hline Urine glucose (\%) & 8.6 & 13.5 & 7.6 & $<0.0001$ & 14 & 7.6 & $<0.0001$ \\
\hline Urine WBCs (\%) & 10.4 & 20.6 & 10.1 & $<0.0001$ & 21.5 & 10 & $<0.0001$ \\
\hline
\end{tabular}

sampling method in recruiting their subjects. They defined "renal failure" as a serum creatinine $>1.8 \mathrm{mg} / \mathrm{dL}$ and reported a prevalence of CKD of $0.79 \%$. However, those subjects with positive proteinuria (by dipstick test) constituted $4.4 \%$ of their population and were not included in their definition of CKD.

The SEEK-India study is similar in design to the KEEP study conducted by the National Kidney Foundation in the USA [19]. It is a convenience cohort, which invokes both strengths and limitations. In a convenience cohort non-random selection of patients may lead to biases that result in screening of "high risk" individuals thereby inflating the prevalence estimates. On the other hand, our prevalence of $17.2 \%$ of CKD is lower to that observed in the NHANES study [20] and is lower than that in the KEEP study [21]. Reports from other Asian countries have shown a high prevalence of CKD. Jafar et al. observed a CKD prevalence of $9 \%$ in men and $11 \%$ women aged 40 years or over (using NKF criteria) [22].

Reports from other countries participating in the Global SEEK project have been published. The results of SEEK-Thailand study using a cluster randomized design

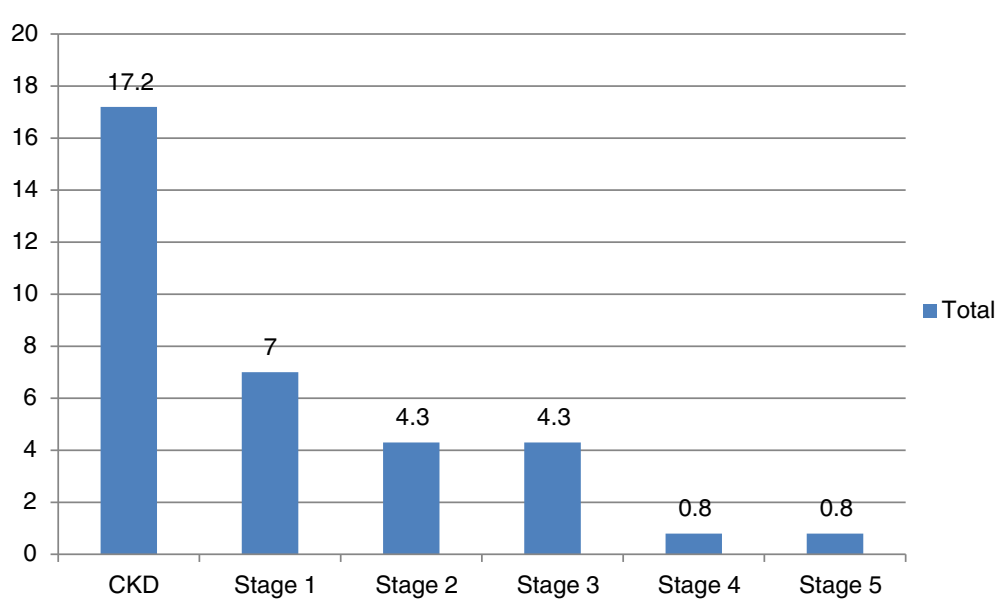

Figure 2 Prevalence of CKD and its stages (using MDRD equation). 


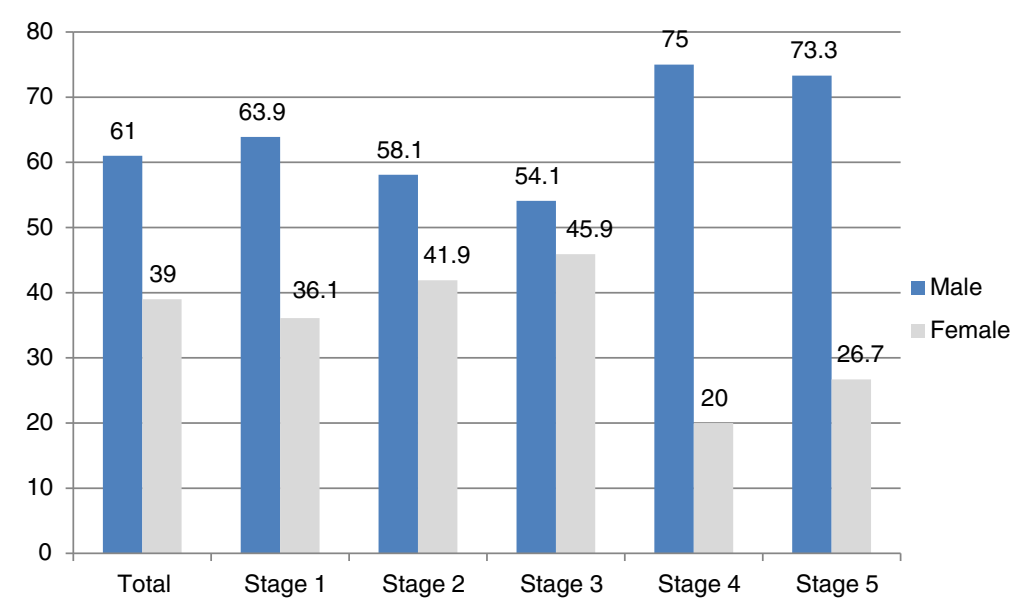

Figure 3 Prevalence of CKD and its stages by gender (using MDRD equation).

showed 17.5\% prevalence of CKD [23]. The SEEK-Saudi Arabia showed a prevalence of 5.7\% [24]. Another report from Thailand has shown an increasing prevalence of decreased kidney function using the criterion of GFR $<60 \mathrm{ml} / \mathrm{min}, 1.7 \%$ in 1985 to $6.8 \%$ in 1997 of the 3499 employees of Electric Generation Authority screened [25]. The prevalence of proteinuria was also increased from $2.64 \%$ and $6.10 \%$ in the same period. Chen et al. have reported a decreased renal function (GFR $<60 \mathrm{ml} / \mathrm{min}$ by the MDRD equation) as $2.53 \%$ of 15,540 Chinese adults (35 to 74 years) [26]. The investigators noted that their study has a limitation in that urinary protein was not measured and persons with albuminuria or microalbuminuria were not included in the estimated prevalence of CKD. Therefore, their findings certainly underestimate the prevalence of CKD in the Chinese adult population. In the present study the prevalence of reduced GFR was $5.9 \%$ of the screened population. The prevalence of earlier stages of CKD primarily indicated by proteinuria was higher and constituted $13.7 \%$. Results from SEEK-Egypt will be published soon.

Increased prevalence of CKD could be partly explained by the high prevalence of risk factors like diabetes and hypertension in the screened population $(18.8 \%$ and $431.1 \%$, respectively). The prevalence of diabetes and hypertension in India varied widely in many studies and ranged from $6-20 \%$ and $13-58 \%$, respectively $[27,28]$. Among the CKD group, $64.5 \%$ had hypertension and $31.6 \%$ had diabetes mellitus. Self reported kidney stones disease was observed in $4.5 \%$.

Despite the high prevalence that we reported in our study, subjects in our cohort had a low awareness of

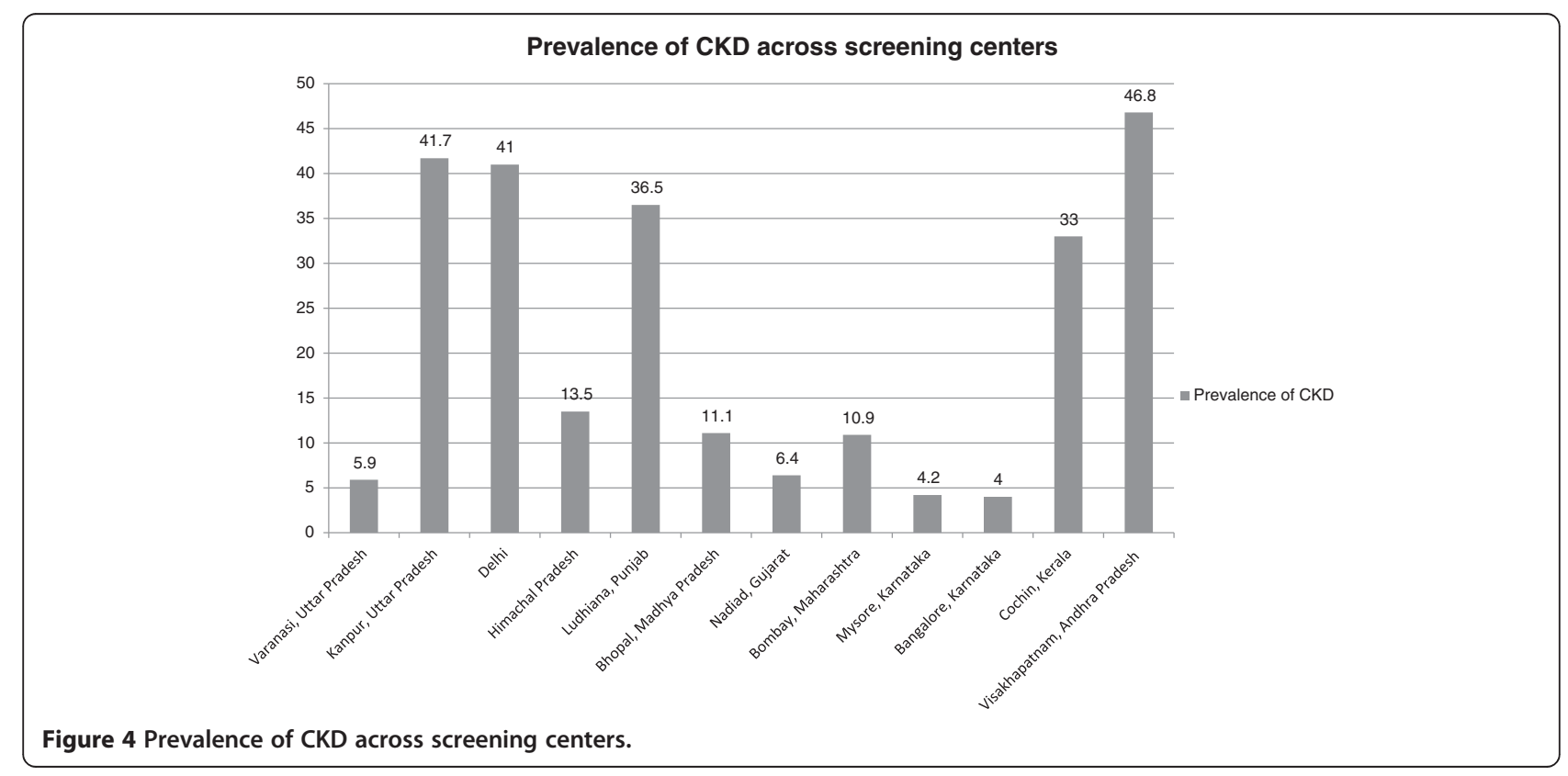




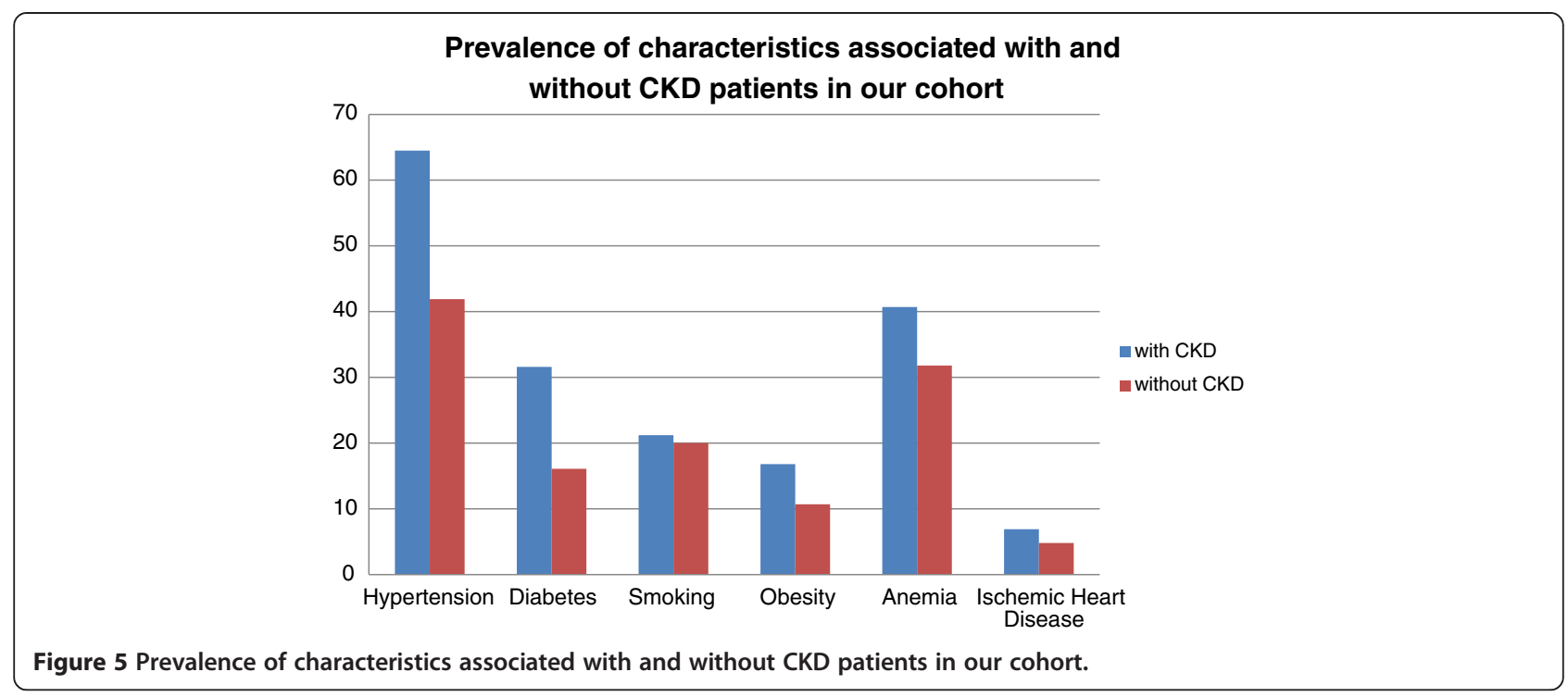

CKD. Only $7.9 \%$ of subjects knew that they had CKD compared to $1.9 \%$ in SEEK-Thailand and $7.1 \%$ in SEEKSaudi Arabia. This might reflect the lack of healthcare resources available to the population. When performing sensitivity analysis using CKD-EPI equation, the prevalence of CKD in our study was $16.4 \%$ (versus $17.2 \%$ using MDRD-3 equation). A recently published study aimed at evaluating the applicability of CKD-EPI equation to eGFR in Chinese patients of different stages of CKD [29], compared it with body surface area-standardized GFR (sGFR), which was measured by diethylenetriaminepentaacetic acid renal dynamic imaging method in 142 CKD cases.

Table 4 Correlations of certain variables with eGFR in the total cohort and in CKD subjects

\begin{tabular}{lccccc}
\hline & \multicolumn{2}{c}{ Total cohort } & & \multicolumn{2}{c}{ CKD subjects } \\
\cline { 2 - 3 } \cline { 5 - 6 } & $\begin{array}{c}\text { Spearman's } \\
\text { rho }\end{array}$ & $\boldsymbol{p}$ & & $\begin{array}{c}\text { Spearman's } \\
\text { rho }\end{array}$ & $\boldsymbol{p}$ \\
\hline Age & -0.454 & $<0.0001$ & & -0.298 & $<0.0001$ \\
Height & 0.0 & 0.993 & & 0.029 & 0.365 \\
Weight & -0.204 & $<0.0001$ & & 0.038 & 0.243 \\
BMI & -0.229 & $<0.0001$ & 0.015 & 0.646 \\
Waist & -0.286 & $<0.0001$ & 0.003 & 0.922 \\
circumference & -0.237 & $<0.0001$ & 0.05 & 0.146 \\
Hip circumference & -0.196 & $<0.0001$ & -0.053 & 0.12 \\
Waist/Hip ratio & -0.236 & $<0.0001$ & -0.196 & $<0.0001$ \\
SBP & -0.159 & $<0.0001$ & -0.118 & $<0.0001$ \\
DBP & -0.061 & $<0.0001$ & 0.263 & $<0.0001$ \\
Hemoglobin & -0.156 & $<0.0001$ & -0.046 & 0.177 \\
$\begin{array}{l}\text { Random blood } \\
\text { glucose }\end{array}$ & & & & \\
Fasting blood & -0.26 & $<0.0001$ & -0.029 & 0.834 \\
glucose & & & & \\
\hline
\end{tabular}

eGFR was positively correlated with sGFR and the average deviation of eGFR from sGFR was $-0.92 \pm 16.36 \mathrm{~mL} / \mathrm{min} /$ $1.73 \mathrm{~m} 2$ ( $\mathrm{p}=0.506)$. They also observed no significant deviation in the CKD from stages 2 to 5 . However, in CKD stage 1 , the deviation was increased with the value of $13.36 \pm 18.44 \mathrm{~mL} / \mathrm{min} / 1.73 \mathrm{~m} \mathrm{[2]}(\mathrm{p}=0.023)$.

In our SEEK-India cohort, the prevalence of CKD was $16.4 \%$. CKD Stage 1 was $8 \%$, slightly higher than the one estimated using MDRD-3 (7\%). However, prevalence of CKD stages 2 and 3 were $3.2 \%$ and $3.3 \%$ respectively, slightly lower than MDRD-3 (4.3\% and 4.3\% respectively). It also showed similar results to MDRD-3 in CKD stages 4 and 5 ( $0.8 \%$ each). Similar comparisons are supported by other studies in UK [30] where it was shown to be more accurate than the MDRD formula at higher GFR and also reduced the estimated prevalence of CKD stages 3-5 by $\sim 0.5 \%-0.7 \%$. Further analysis of KEEP study showed similar results $[31,32]$ and the KEEP investigators stated that CKD-EPI will be used to report eGFR in KEEP.

Sixty three percent of our total sample, and almost half of the CKD group (52.6\%), earns $<\$ 125$ a month. Poverty and lack of education often go together. Among the CKD population, $37.6 \%$ had less than high school which may have contributed to this lack of awareness. In the NHANES study $11.6 \%$ of men and $5.5 \%$ of women in CKD stage-3 knew about their disease [20].

Our study had several potential limitations. We used a convenience study design rather than a cluster randomization design and/or household surveys. We mirrored our design on that used by the NKF KEEP cohort in order to bench mark our community based cohort with the results of KEEP. However our sampling strategy may not be ideal for evaluation of true prevalence. Another limitation was the single measurement of serum creatinine and urine albumin. Repeated measures might 
improve the precision around our reporting of the data. Furthermore, a subsequent measurement after 3 months might have provided additional insights into the chronicity of the disease. Additionally, the prevalence of CKD might have been overestimated by using the Bayer's multistix 10 which detects urine protein, not albuminuria. Another limitation is that we used the MDRD equation using the race factor for compatible for Americans. However, there are concerns of the application of the definition and staging system for current eGFR estimating equations to the Indian population. Different diet and muscle mass in the Indian as compared to the North American populations may lead to both differences in the normal level for kidney function in the population as well as the relationship between creatinine and GFR as reflected in the estimating equations; where these equations have been predominantly developed and validated.

\section{Conclusion}

In conclusion, in this large, community-based crosssectional study using a convenience sample of SEEK, we successfully carried out CKD screening using simple tests to estimate e-GFR and protienuria. The prevalence of CKD was observed to be $17.2 \%$ with $\sim 6 \%$ have CKD stage 3 or worse. CKD risk factors were similar to those reported in earlier studies. Awareness was observed to be low. This data supports the importance of improving the education and early detection of CKD. It should be stressed to all primary care physicians taking care of hypertensive and diabetic patients to screen for early kidney damage. Early intervention may retard the progression of kidney disease. Planning for the preventive health policies and allocation of more resources for the treatment of CKD/ESRD patients are imperative in India.

\section{Additional file}

Additional file 1: SEEK Project - Screening Questionnaire.

\section{Competing interests}

The authors declare that they have no competing interest.

\section{Authors' contributions}

BVM and AKS - conceived and designed the study. AKS - acquired funding, supervised the overall execution of the study. BVM - cleaned the database. YMKF - analyzed and interpreted the data. YMKF, BVM, KKS and AKS - wrote the manuscript. All other co-authors - conducted and supervised the study procedures and the operational execution in the local Indian centers. All authors read and approved the final manuscript.

\section{Acknowledgement}

The study was supported by grant from Jansen-Cilag. The sponsor had no role in study design, data collection, data analysis or writing the manuscript. The SEEK study investigators express their appreciation to Janssen-Cilag India (Kuldeep Pandit and Bhushan Akshikar) and Johnson and Johnson, USA (Dr. Suresh Aravind). We are also grateful to the Indian Society of Nephrology for their strong support of this project. The authors would like to thank Dania Daye, MD-PhD student and HHMI-NIBIB* Interfaces Scholar at the University of Pennsylvania School of Medicine, for her instrumental contribution in the CKD-EPI eGFR equation results. *HHMI: Howard Hughes Medical Institute, NIBIB: National Institute of Biomedical Imaging and Bioengineering.

\section{Author details}

'Brigham \& Women's Hospital \& Harvard Medical School, 75 Francis Street, Boston, MA 02115, USA. ${ }^{2}$ NKF, Mumbai, India. ${ }^{3}$ Hinduja Hospital, Mumbai, India. ${ }^{4}$ Vivekananda Memorial Hospital, H.D.Kote, Saragur, Mysore, India.

${ }^{5}$ Manipal Institute of Nephrology \& Urology, Bangalore, India. ${ }^{6}$ Deep Hospital, Ludhiana, India. ${ }^{7}$ Indraprastha Apollo Hospital, New Delhi, India. ${ }^{8}$ Bombay Hospital, Mumbai, India. ${ }^{9}$ Fortis Flt. Lt. Rajan Dhall Hospital, New Delhi, India. ${ }^{10}$ Bhopal Memorial Hospital \& Research Center, Bhopal, India. ${ }^{11}$ PVS Memorial Hospital, Cochin, India. ${ }^{12}$ Institute of Medical Sciences, BHU, Varanasi, India. ${ }^{13}$ Muljibhai Patel Urological Hospital, Nadiad, India. ${ }^{14}$ Sir Ganga Ram Hospital, New Delhi, India. ${ }^{15}$ Wockhardt Hospitals, Bangalore, India. ${ }^{16}$ Kanpur Rotary Kidney Foundation, Kanpur, India. ${ }^{17}$ Lilavati Hospital, Mumbai, India. ${ }^{18}$ Dr. R.M. Lohia Hospital, New Delhi, India. ${ }^{19}$ Sanjay Gandhi Postgraduate Institute of Medical Sciences, Lucknow, India. ${ }^{20}$ Andhra Medical College \& King George Hospital, Vishakhapatanam, India.

Received: 31 May 2012 Accepted: 20 March 2013

Published: 28 May 2013

\section{References}

1. Ruggenenti P, Schieppati A, Remuzzi G: Progression, remission, regression of chronic renal diseases. Lancet 2001, 357(9268):1601-8.

2. Wild S, Roglic G, Green A, Sicree R, King H: Global prevalence of diabetes: estimates for the year 2000 and projections for 2030. Diabetes Care 2004 27(5):1047-53.

3. Gupta R: Trends in hypertension epidemiology in India. J Hum Hypertens 2004, 18(2):73-8.

4. Anand MP: Prevalence of hypertension amongst Mumbai executives. J Assoc Physicians India 2000, 48(12):1200-1.

5. Modi GK, Jha V: The incidence of end-stage renal disease in India: a population-based study. Kidney Int 2006, 70(12):2131-3.

6. Kher V: End-stage renal disease in developing countries. Kidney Int 2002, 62(1):350-62.

7. Jha V: End-stage renal care in developing countries: the india experience. Ren Fail 2004, 26(3):201-8.

8. Sakhuja V, Sud K: End-stage renal disease in India and Pakistan: burden of disease and management issues. Kidney Int Suppl 2003, 83(83):S115-8.

9. Pickering TG, Hall JE, Appel LJ, Falkner BE, Graves JW, Hill MN, et al: Recommendations for blood pressure measurement in humans: an AHA scientific statement from the council on high blood pressure research professional and public education subcommittee. J Clin Hypertens (Greenwich) 2005, 7(2):102-9.

10. Levey AS, Coresh J, Greene T, Marsh J, Stevens LA, Kusek JW, et al: Expressing the modification of diet in renal disease study equation for estimating glomerular filtration rate with standardized serum creatinine values. Clin Chem 2007, 53(4):766-72.

11. Chobanian AV, Bakris GL, Black HR, Cushman WC, Green LA, Izzo JL Jr, et al: The seventh report of the joint national committee on prevention, detection, evaluation, and treatment of high blood pressure: the JNC 7 report. JAMA 2003, 289(19):2560-72

12. Expert Committee on the Diagnosis and Classification of Diabetes Mellitus: Report of the expert committee on the diagnosis and classification of diabetes mellitus. Diabetes Care 2003, 26(Suppl 1):S5-20.

13. Alberti KG, Zimmet P, Shaw J, IDF Epidemiology Task Force Consensus Group: The metabolic syndrome-a new worldwide definition. Lancet 2005, 366(9491):1059-62.

14. Vassalotti JA, Stevens LA, Levey AS: Testing for chronic kidney disease: a position statement from the national kidney foundation. Am J Kidney Dis 2007, 50(2):169-80.

15. Levey AS, Stevens LA, Schmid CH, Zhang YL, Castro AF 3rd, Feldman HI: A new equation to estimate glomerular filtration rate. Ann Intern Med 2009, 150(9):604-12

16. Mani MK: Experience with a program for prevention of chronic renal failure in india. Kidney Int Supp/ 2005, 94(94):S75-8. 
17. Mani MK: Prevention of chronic renal failure at the community level. Kidney Int Supp/ 2003, 83(83):S86-9.

18. Agarwal SK, Dash SC, Irshad M, Raju S, Singh R, Pandey RM: Prevalence of chronic renal failure in adults in Delhi, India. Nephrol Dial Transplant 2005, 20(8):1638-42.

19. Brown WW, Peters RM, Ohmit SE, Keane WF, Collins A, Chen SC, et al: Early detection of kidney disease in community settings: the kidney early evaluation program (KEEP). Am J Kidney Dis 2003, 42(1):22-35.

20. Coresh J, Selvin E, Stevens LA, Manzi J, Kusek JW, Eggers P, et al: Prevalence of chronic kidney disease in the United States. JAMA 2007, 298(17):2038-47.

21. Jurkovitz CT, Qiu Y, Wang C, Gilbertson DT, Brown WW: The kidney early evaluation program (KEEP): program design and demographic characteristics of the population. Am J Kidney Dis 2008, 51 (4 Suppl 2):S3-12.

22. Jafar TH: Hypertension and kidney disease in Asia. Curr Opin Nephrol Hypertens 2006, 15(3):291-5.

23. Ingsathit A, Thakkinstian A, Chaiprasert A, Sangthawan P, Gojaseni P, Kiattisunthorn $\mathrm{K}$, et al: Prevalence and risk factors of chronic kidney disease in the thai adult population: Thai SEEK study. Nephrol Dial Transplant 2010, 25(5):1567-75.

24. Alsuwaida AO, Farag YM, Al Sayyari AA, Mousa D, Alhejaili F, Al-Harbi A, et al: Epidemiology of chronic kidney disease in the kingdom of saudi arabia (SEEK-saudi investigators) - a pilot study. Saudi J Kidney Dis Transp/ 2010, 21(6):1066-72.

25. Domrongkitchaiporn S, Sritara P, Kitiyakara C, Stitchantrakul W, Krittaphol V, Lolekha P, et al: Risk factors for development of decreased kidney function in a Southeast Asian population: a 12-year cohort study. J Am Soc Nephrol 2005, 16(3):791-9.

26. Chen J, Wildman RP, Gu D, Kusek JW, Spruill M, Reynolds K, et al: Prevalence of decreased kidney function in Chinese adults aged 35 to 74 years. Kidney Int 2005, 68(6):2837-45.

27. Prasad DS, Kabir Z, Dash AK, et al: Prevalence and risk factors for diabetes and impaired glucose tolerance in Asian Indians: a community survey from urban Eastern India. Diabetes Metab Syndr 2012, 6(2):96-101. doi:10.1016/j.dsx.2012.05.016. Epub 2012

28. Devi P, Rao M, Sigamani A, et al: Prevalence, risk factors and awareness of hypertension in India: a systematic review. J Hum Hypertens 2012. doi:10.1038/jhh.2012.33.

29. Du X, Hu B, Jiang L, Wan X, Fan L, Wang F, et al: Implication of CKD-EPI equation to estimate glomerular filtration rate in Chinese patients with chronic kidney disease. Ren Fail 2011, 33(9):859-65.

30. Gifford FJ, Methven S, Boag DE, Spalding EM, Macgregor MS: Chronic kidney disease prevalence and secular trends in a UK population: the impact of MDRD and CKD-EPI formulae. QJM 2011, 104(12):1045-53.

31. Stevens LA, Li S, Kurella Tamura M, Chen SC, Vassalotti JA, Norris KC, et al: Comparison of the CKD epidemiology collaboration (CKD-EPI) and modification of diet in renal disease (MDRD) study equations: risk factors for and complications of CKD and mortality in the kidney early evaluation program (KEEP). Am J Kidney Dis 2011, 57(3 Suppl 2):S9-16.

32. McFarlane SI, McCullough PA, Sowers JR, Soe K, Chen SC, Li S, et al: Comparison of the CKD epidemiology collaboration (CKD-EPI) and modification of diet in renal disease (MDRD) study equations: prevalence of and risk factors for diabetes mellitus in CKD in the kidney early evaluation program (KEEP). Am J Kidney Dis 2011, 57(3 Suppl 2):S24-31.

doi:10.1186/1471-2369-14-114

Cite this article as: Singh et al:: Epidemiology and risk factors of chronic kidney disease in India - results from the SEEK (Screening and Early Evaluation of Kidney Disease) study. BMC Nephrology 2013 14:114. 